INPLASY

PROTOCOL

To cite: Cheng et al. Efficacy of intra-arterial chemotherapy plus intravesical installation for high-risk non-muscle-invasive bladder cancer (NMIBC): a meta-analysis. Inplasy protocol 202110031. doi:

10.37766/inplasy2021.1.0031

Received: 10 January 2021

Published: 11 January 2021

Corresponding author: Cheng Chunliang

chengchunliang1@163.com

Author Affiliation:

Department of Urology, Xiangya Hospital, Central South University, NO.87

Xiangya Road, Changsha, Hunan 41000, P.R. China

Support: Xiangya Hospital.

Review Stage at time of this submission: Data analysis.

Conflicts of interest:

None.

\section{Efficacy of intra-arterial chemotherapy plus intravesical installation for high-risk non- muscle-invasive bladder cancer (NMIBC): a meta-analysis}

Cheng, C1; Qiu, D².

Review question / Objective: (I) population (P): patients pathologically diagnosed NMIBC after transurethral resection of bladder tumor; (II) intervention (I): patients treated with intravescial chemotherapy plus intravesical chemotherapy; (III) comparison (C): treated with intravesical chemotherapy alone; (IV) outcomes (O): OS (periods from the start of treatment to death from any cause), cancer-specific survival (CSS) (cancer survival in the absence of other causes of death), progression-free survival (PFS) (periods from the start of treatment to disease progression or death from any cause), tumor recurrence rate, tumor progression rate or tumorspecific-death rate (V) study design (S): any studies related to this subject including prospective or retrospective studies or randomized controlled trial.

INPLASY registration number: This protocol was registered with the International Platform of Registered Systematic Review and Meta-Analysis Protocols (INPLASY) on 11 January 2021 and was last updated on 11 January 2021 (registration number INPLASY202110031).

\section{INTRODUCTION}

Review question / Objective: (I) population $(P)$ : patients pathologically diagnosed NMIBC after transurethral resection of bladder tumor; (II) intervention (I): patients treated with intravescial chemotherapy plus intravesical chemotherapy; (III) comparison (C): treated with intravesical chemotherapy alone; (IV) outcomes (O): OS (periods from the start of treatment to death from any cause), cancer-specific survival (CSS) (cancer survival in the absence of other causes of death), 
progression-free survival (PFS) (periods from the start of treatment to disease progression or death from any cause), tumor recurrence rate, tumor progression rate or tumor-specific-death rate (V) study design (S): any studies related to this subject including prospective or retrospective studies or randomized controlled trial.

Condition being studied: The high-risk nonmuscle-invasive bladder cancer (NMIBC) has a high risk of recurrence and progression after transurethral resection(TUR). It is common to use adjuvant intravesical treatment after TUR. Adjuvant intravesical therapy with chemotherapy may help to prevent recurrence but not progression. While adjuvant intravesical therapy with BCG not only prevents recurrence but also prevents, or at least delays, the risk of tumour progression with a limitation of complications at different rates. Furthermore, BCG has not been approved for clinical use by the Food and Drug Administration in China. Thus, the search new treatment strategies for patients is now necessary. Combining intra-arterial chemotherapy with intravesical chemotherapy after TUR is a novel treatment strategy for NMIBC which need to be explore its efficacy.

\section{METHODS}

Search strategy: I(()((((bladder cancer) OR (non-muscle invasive)) OR (Superficial)) OR (Early)) OR (Ta)) OR (T1)) OR (Tis)) OR (CIS)) AND (()((intra-arterial chemotherapy) OR (Intra-arterial infusion)) OR (Intra-arterial therapy)) OR (Intra-arterial injection)) OR (gemcitabine)) OR (cisplatin))) AND ( (()(()(Intravesical perfusion) OR (Intravesical chemotherapy)) OR (Intravesical therapy)) OR (Intravesical irrigation)) OR (BCG)) OR (Bacille CalmetteGuérin)) OR (epirubicin)) OR (pirarubicin)).

Participant or population: Patients with high-risk non-muscle-invasive bladder cancer.
Intervention: Intra-arterial chemotherapy combined with intravesical chemotherapy.

Comparator: Intravesical chemotherapy alone.

Study designs to be included: Randomized controlled trial or retrospective or prospective study.

Eligibility criteria: (1) patients underwent bladder-preserving surgery (2) pathologically confirmed NMIBC (3) interventions were intravescial chemotherapy plus intravesical chemotherapy (4) sufficient data to extract including tumor recurrence rate, tumor progression rate, overall survival, cancerspecific survival or progression-free survival (5) prospective or retrospective studies or randomized controlled trial (6) published with English language.

Information sources: Pubmed and Embase and Cochrane Library.

Main outcome(s): Tumor recurrence rate, tumor progression rate, tumor-specific death rate and overall survival.

Additional outcome(s): Adverse events.

Quality assessment / Risk of bias analysis: GRADE quality assessments and funnel plot.

Strategy of data synthesis: Two reviewers were enrolled to assess the 6 eligible publications and extract the data independently. Any conflicts would resort to the judgement by the third reviewer. The detailed data we extracted included the authors' name, publication year, country, study design, patient number, gender number, age, tumor stage, IAC and IVC regimen, duration of follow-up, event number of the intervention and comparison group and oncologic outcomes.

Subgroup analysis: subgroup 1: studies involving patients received IAC (cisplatin+epirubicin )+IVI (pirarubicin) Vs. studies with other IAC +IVC regime 
subgroup 2: studies involving patients underwent BCG based IVI Vs. studies involving IVI no matter BCG or chemotherapy.

Sensibility analysis: Sensitivity analysis will be performed by eliminating one study at a time.

Language: English.

Country(ies) involved: mainland China.

Keywords: intra-arterial chemotherapy; Intravesical installation; high-risk nonmuscle-invasive bladder cancer; metaanalysis.

Contributions of each author:

Author 1 - Cheng Chunliang.

Email: chengchunliang1@163.com

Author 2 - Qiu Dongxu.

Email: zuxbxyyy@126.com 